\title{
Generalized proportional fractional integral functional bounds in Minkowski's inequalities
}

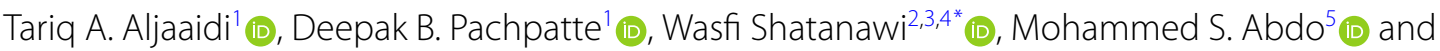
Kamaleldin Abodayeh²

\section{"Correspondence:}

wshatanawi@psu.edu.sa

${ }^{2}$ Department of Mathematics and

General Sciences, Prince Sultan

University, Riyadh, Saudi Arabia

${ }^{3}$ Department of Medical Research,

China Medical University Hospital,

China Medical University, Taichung,

40402, Taiwan

Full list of author information is

available at the end of the article

\section{Springer}

\begin{abstract}
In this research paper, we improve some fractional integral inequalities of Minkowski-type. Precisely, we use a proportional fractional integral operator with respect to another strictly increasing continuous function $\psi$. The functions used in this work are bounded by two positive functions to get reverse Minkowski inequalities in a new sense. Moreover, we introduce new fractional integral inequalities which have a close relationship to the reverse Minkowski-type inequalities via $\psi$-proportional fractional integral, then with the help of this fractional integral operator, we discuss some new special cases of reverse Minkowski-type inequalities through this work. An open issue is covered in the conclusion section to extend the current findings to be more general.
\end{abstract}

MSC: 26D10;26A33

Keywords: Minkowski inequalities; Fractional inequalities; $\psi$-proportional fractional operators

\section{Introduction}

During their uncompromising effort in the expansion of mathematics, mathematicians have recently expanded the traditional calculus of derivatives and integrals for integer orders to the generalized form of conventional derivatives and integrals of noninteger order, these noninteger-order derivatives/integrals are referred to as fractional calculus, which during a few previous decades became one of very influential branches of mathematics, especially, when dealing with the differential/integral equations and inequalities.

The fractional calculus theory became important due to its significant applications in several areas such as physics, fluid dynamics, control theory, computer networking, signal processing, biology, image processing, and other areas. One of the common tools of researchers is the use of fractional derivative/integral operators and, consequently, numerous distinct fractional derivatives/integrals have been introduced, such as the RiemannLiouville, Liouville, Weyl-type, Katugampola, Hadamard, Atangana-Baleanu, proportional fractional integral, and some other kinds, which can be found in the monograph of Kilbas et al. [1]. In [2], the authors presented very diverse properties in the framework of generalized fractional operators with respect to another function. In addition, these opera-

(c) The Author(s) 2021. This article is licensed under a Creative Commons Attribution 4.0 International License, which permits use sharing, adaptation, distribution and reproduction in any medium or format, as long as you give appropriate credit to the original author(s) and the source, provide a link to the Creative Commons licence, and indicate if changes were made. The images or other third party material in this article are included in the article's Creative Commons licence, unless indicated otherwise in a credit line to the material. If material is not included in the article's Creative Commons licence and your intended use is not permitted by statutory regulation or exceeds the permitted use, you will need to obtain permission directly from the copyright holder. To view a copy of this licence, visit http://creativecommons.org/licenses/by/4.0/. 
tors were used to perform qualitative analysis of fractional differential equations and some epidemiological models, see [3-10]. Numerical analysis of fractional differential equations and some new concepts in fractional calculus have been extensively investigated recently, and a variety of interesting results on the topic have been established; for instance, see [1113]. On the other hand, we refer here to some works related to the proportional fractional operator that we will work on; see [14-17].

No one can deny the crucial importance of inequalities in mathematics; in particular, in classical integral and differential equations, they play a meaningful role. Therefore, not too long ago, many helpful and remarkable mathematical inequalities were introduced by several authors. One mathematical inequality which has wide applications was given by Hermann Minkowski. This inequality, in the last few decades, has received considerable attention from several researchers, and many articles have appeared in the literature. In (2006), Bougoffa presented the classical integral version of Minkowski inequality as follows:

Theorem 1.1 ([18]) Consider positive functions $\eta, \varsigma$ in $L^{z}[s, \omega]$ with $z \in[1, \infty)$. Assume that $0<q \leq \frac{\eta(\kappa)}{\zeta^{(}(\kappa)} \leq Q, \kappa \in[s, \omega]$, where $q, Q \in \mathbb{R}_{+}^{*}$. Then we have

$$
\left(\int_{s}^{\omega} \eta^{z}(\omega) d \omega\right)^{\frac{1}{z}}+\left(\int_{s}^{\omega} \varsigma^{z}(\omega) d \omega\right)^{\frac{1}{z}} \leq \frac{(2+q) Q+1}{(1+Q)(1+q)}\left(\int_{s}^{\omega}(\eta+\varsigma)^{z}(\omega) d \omega\right)^{\frac{1}{z}}
$$

In the same work, the author gave also the following inequality:

Theorem 1.2 ([18]) For positive functions $\eta, \varsigma$ in $L^{z}[s, \omega]$ with $z \in[1, \infty)$, assume that $0<q \leq \frac{\eta(\kappa)}{5(\kappa)} \leq Q, \kappa \in[s, \omega]$, where $q, Q \in \mathbb{R}_{+}^{*}$. Then we have

$$
\begin{aligned}
& \left(\int_{s}^{\omega} \eta^{z}(\omega) d \omega\right)^{\frac{2}{z}}+\left(\int_{s}^{\omega} \varsigma^{z}(\omega) d \omega\right)^{\frac{2}{z}} \\
& \quad \geq\left(\frac{(1+Q)(1+q)}{Q}-2\right)\left(\int_{s}^{\omega} \eta^{z}(\omega) d \omega\right)^{\frac{1}{z}}\left(\int_{s}^{\omega} \varsigma^{z}(\omega) d \omega\right)^{\frac{1}{z}} .
\end{aligned}
$$

The fractional integral version of the reverse Minkowski inequality with constants bounds was given in 2010 by Dahmani [19], and in same work he also gave the fractional integral version of (1.2) as follows:

Theorem 1.3 Let $\theta>0, z \geq 1$. For positive functions $\eta, \varsigma$ on $[0, \infty)$, we have for all $\omega>0$, and $\mathcal{I}^{\theta} \eta^{z}(\omega), \mathcal{I}^{\theta} \varsigma^{z}(\omega)<\infty$, that if $0<q \leq \frac{\eta(\kappa)}{\varsigma(\kappa)} \leq Q, \kappa \in[0, \omega]$, then

$$
\left(\mathcal{I}^{\theta} \eta^{z}(\omega) d \omega\right)^{\frac{1}{z}}+\left(\mathcal{I}^{\theta} \varsigma^{z}(\omega) d \omega\right)^{\frac{1}{z}} \leq \frac{(2+q) Q+1}{(1+Q)(1+q)}\left(\mathcal{I}^{\theta}(\eta+\varsigma)^{z}(\omega) d \omega\right)^{\frac{1}{z}}
$$

Theorem 1.4 Let $\theta>0, z \geq 1$. For positive functions $\eta, \varsigma$ on $[0, \infty)$, we have for all $\omega>0$, and $\mathcal{I}^{\theta} \eta^{z}(\omega), \mathcal{I}^{\theta} \varsigma^{z}(\omega)<\infty$, that if $0<q \leq \frac{\eta(\kappa)}{\zeta(\kappa)} \leq Q, \kappa \in[0, \omega]$, then

$$
\begin{aligned}
& \left(\mathcal{I}^{\theta} \eta^{z}(\omega) d \omega\right)^{\frac{2}{z}}+\left(\mathcal{I}^{\theta} \varsigma^{z}(\omega) d \omega\right)^{\frac{2}{z}} \\
& \quad \geq\left(\frac{(1+Q)(1+q)}{Q}-2\right)\left(\mathcal{I}^{\theta} \eta^{z}(\omega) d \omega\right)^{\frac{1}{z}}\left(\mathcal{I}^{\theta} \varsigma^{z}(\omega) d \omega\right)^{\frac{1}{z}} .
\end{aligned}
$$


Erhan et al. [20] in 2010 presented reverse Minkowski and Hermite-Hadamard inequalities involving two functions for the classical Riemann integral. In 2012, Yang [21] introduced a new fractional quantum integral Minkowski inequality and other inequalities employing a fractional $q$-integral on the specific time scale. In 2013, Taf with Brahim [22] and Chinchane with Pachpatte [23] used the Hadamard fractional integral operator to establish the reverse Minkowski's inequality. Vanterler et al. [24] in 2018 employed Katugampola fractional integral operator to establish reverse Minkowski inequality and other related inequalities. In 2019, Rahman et al. [25] presented the reverse Minkowski's inequality, and they also established some other fractional integral inequalities by using generalized proportional fractional integral operators. In 2020, the reverse Minkowski inequalities were studied by Aljaaidi and Pachpatte [26] via the Riemann-Liouville operator with respect to the positive monotone function $\varphi$. In the same year, Rashid et al. [27] presented a note on reverse Minkowski inequalities by using a generalized proportional fractional operator involving another function. It should be noted that in that year also Maga et al. [28] employed Mittag-Leffler function with the corresponding fractional integral to introduce new Minkowski-type inequalities. For more about some recent and earlier results related to the Minkowski inequalities, we point the readers to [29-36].

The fractional integral reverse Minkowski inequality with functional bounds is given by Aljaaidi and Pachpatte [37] by employing the Riemann-Liouville operator as follows:

Theorem 1.5 ([37]) Consider positive functions $\eta$, $\varsigma$ on $[0, \infty)$ with $\mathcal{I}^{\theta} \eta^{z}(\omega), \mathcal{I}^{\theta} \varsigma^{z}(\omega)<\infty$, $\forall \omega \in[0, \infty)$ and for all $\theta>0, z \geq 1$. Let $\psi$ be a positive and strictly increasing continuous function. Assume that $\mathrm{七}, \varphi$ are positive functions such that $0<\mathrm{七}(\varepsilon) \leq \frac{\eta(\kappa)}{\zeta(\kappa)} \leq \varphi(\varepsilon), \kappa, \varepsilon \in$ $[0, \omega]$. Then we have

$$
\begin{aligned}
& {\left[\mathcal{I}^{\theta} \eta^{z}(\omega)\right]^{\frac{1}{z}}+\left[\mathcal{I}^{\theta} \varsigma^{z}(\omega)\right]^{\frac{1}{z}}} \\
& \quad \leq \frac{\Gamma(\theta+1)}{\omega^{\theta}}\left\{\mathcal{I}^{\theta}\left(\frac{\varphi(\omega)}{\varphi(\omega)+1}\right)+\mathcal{I}^{\theta}\left(\frac{1}{\mathrm{E}(\omega)+1}\right)\right\}\left[\mathcal{I}^{\theta}(\eta+\varsigma)^{z}(\omega)\right]^{\frac{1}{z}} .
\end{aligned}
$$

In the same work, the authors gave also the following inequality:

Theorem 1.6 ([37]) Consider positive functions $\eta, \varsigma$ on $[0, \infty)$ with $\mathcal{I}^{\theta} \eta^{z}(\omega), \mathcal{I}^{\theta} \varsigma^{z}(\omega)<\infty$, $\forall \omega \in[0, \infty)$ and for all $\theta>0, z \geq 1$. Let $\psi$ be a positive and strictly increasing continuous function. Assume that $\mathrm{七}, \varphi$ are positive functions such that $0<\mathrm{七}(\varepsilon) \leq \frac{\eta(\kappa)}{\zeta(\kappa)} \leq \varphi(\varepsilon), \kappa, \varepsilon \in$ $[0, \omega]$. Then we have

$$
\begin{aligned}
& {\left[\mathcal{I}^{\theta} \eta^{z}(\omega)\right]^{\frac{2}{z}}+\left[\mathcal{I}^{\theta} \varsigma^{z}(\omega)\right]^{\frac{2}{z}}} \\
& \quad \geq\left\{\frac{\Gamma^{2}(\theta+1)}{\omega^{2 \theta}} \mathcal{I}^{\theta}\left(\frac{\varphi(\omega)+1}{\varphi(\omega)}\right) \mathcal{I}^{\theta}(\mathrm{L}(\omega)+1)-2\right\}\left[\mathcal{I}^{\theta} \eta^{z}(\omega)\right]^{\frac{1}{z}}\left[\mathcal{I}^{\theta} \varsigma^{z}(\omega)\right]^{\frac{1}{z}} .
\end{aligned}
$$

Motivated by the results mentioned above, in particular, Theorems 1.5 and 1.6, we strive in our recent work to employ a generalized proportional fractional integral with respect to a strictly increasing continuous function $\psi$ to establish the functional bounds in the reverse Minkowski type inequalities in terms of the fractional integral. Moreover, we introduce new functional bounds in fractional inequalities which are related to the reverse Minkowski's inequality employing the $\psi$-proportional fractional operator. During this 
work, we will enrich the research by discussing some special cases related to the current work.

We organized this paper as follows: in Sect. 2, we invoke some definitions, results, notations, and precursory information which we will employ throughout this work. Section 3 is devoted to essential results of reverse Minkowski's inequalities involving functional bounds. In Sect. 4, we give some other related results which include proportional fractional integral with respect to another strictly increasing continuous function.

\section{Essential preliminaries}

Here, we present some elementary definitions and properties of some basic fractional integral operators and present the fractional integral operator utilized to obtain our new results.

Definition 2.1 ([1]) Consider an integrable function $\eta$ on $[s, t]$ and let $s \geq 0$. We define, for all $\theta>0$,

$$
\mathcal{I}_{s^{+}}^{\theta} \eta(\omega)=\frac{1}{\Gamma(\theta)} \int_{s}^{\omega}(\omega-\kappa)^{\theta-1} \eta(\kappa) d \kappa, \quad \kappa>s
$$

and

$$
\mathcal{I}_{t^{-}}^{\theta} \eta(\omega)=\frac{1}{\Gamma(\theta)} \int_{\omega}^{t}(\kappa-\omega)^{\theta-1} \eta(\kappa) d \kappa, \quad \omega<t
$$

where $\Gamma(\theta)=\int_{0}^{\infty} e^{-x} x^{\delta-1} d x$ is the Gamma function and $\mathcal{I}_{s^{+}}^{0} \eta(\omega)=\mathcal{I}_{t^{-}}^{0} \eta(\omega)=\eta(\omega) ; \mathcal{I}_{s^{+}}^{\theta}$ and $\mathcal{I}_{t^{-}}^{\theta}$ are called the left- and right-sided Riemann-Liouville fractional integrals, respectively.

Definition 2.2 ([1,38]) For an integrable function $\eta$ on the interval $\Upsilon$ and for an increasing function $\psi$, where $\psi(\omega) \in C^{1}(\Upsilon, \mathbb{R})$ is such that $\psi^{\prime}(\omega) \neq 0, \omega \in \Upsilon$, we define, for all $\theta>0$,

$$
\mathcal{I}_{s^{+}}^{\theta ; \psi} \eta(\omega)=\frac{1}{\Gamma(\theta)} \int_{s}^{\omega} \psi^{\prime}(\kappa)[\psi(\omega)-\psi(\kappa)]^{\theta-1} \eta(\kappa) d \kappa
$$

and

$$
\mathcal{I}_{t^{-}}^{\theta ; \psi} \eta(\omega)=\frac{1}{\Gamma(\theta)} \int_{\omega}^{t} \psi^{\prime}(\kappa)[\psi(\kappa)-\psi(\omega)]^{\theta-1} \eta(\kappa) d \kappa,
$$

where $\mathcal{I}_{s^{+}}^{\theta ; \psi} \eta(\omega)$ and $\mathcal{I}_{t^{-}}^{\theta ; \psi} \eta(\omega)$ are called the left- and right-sided $\psi$-Riemann-Liouville fractional integrals of a function $\eta$, respectively.

Definition 2.3 ([39]) Consider an integrable function $\eta$ and let $v>0$. We define, for all $\theta \in \mathbb{C}, \operatorname{Re}(\theta) \geq 0$,

$$
\begin{aligned}
\left({ }_{s} D^{\theta, v} \eta\right)(\omega) & =D^{m, v}{ }_{s} \mathcal{I}^{m-\theta, v} \eta(\omega) \\
& =\frac{D_{\omega}^{m, v}}{v^{m-\theta} \Gamma(m-\theta)} \int_{s}^{\omega} \exp \left[\frac{v-1}{v}(\omega-\kappa)\right](\omega-\kappa)^{m-\theta-1} \eta(\kappa) d \kappa
\end{aligned}
$$


and

$$
\begin{aligned}
\left(D_{t}^{\theta, v} \eta\right)(\omega) & ={ }_{\gamma} D^{m, v} \mathcal{I}_{t}^{m-\theta, v} \eta(\omega) \\
& =\frac{\gamma D_{\omega}^{m, v}}{v^{m-\theta} \Gamma(m-\theta)} \int_{\omega}^{t} \exp \left[\frac{v-1}{v}(\kappa-\omega)\right](\kappa-\omega)^{m-\theta-1} \eta(\kappa) d \kappa
\end{aligned}
$$

where

$$
D^{m, v}=\underbrace{D^{v} D^{v} \cdots D^{v}}_{m \text {-times }}, \quad m=[\operatorname{Re}(\theta)]+1,
$$

and

$$
\left({ }_{\gamma} D^{v} \eta\right)(\omega)=(1-v) \eta(\omega)-v \eta^{\prime}(\omega), \quad{ }_{\gamma} D^{m, v}=\underbrace{{ }_{\gamma} D_{\gamma}^{v} D^{v} \cdots{ }_{\gamma} D^{v}}_{m \text {-times }} ;
$$

$\left.{ }_{s} D^{\theta, v} \eta\right)(\omega)$ and $\left(D_{t}^{\theta, v} \eta\right)(\omega)$ are called the left- and right-sided proportional fractional derivatives of a function $\eta$ of $\operatorname{order} \theta$, respectively.

Definition 2.4 ([39]) Consider an integrable function $\eta$ and let $v>0$. We define, for all $\theta \in \mathbb{C}, \operatorname{Re}(\theta) \geq 0$,

$$
\left({ }_{s} \mathcal{I}^{\theta, v} \eta\right)(\omega)=\frac{1}{v^{\theta} \Gamma(\theta)} \int_{s}^{\omega} \exp \left[\frac{v-1}{v}(\omega-\kappa)\right](\omega-\kappa)^{\theta-1} \eta(\kappa) d \kappa
$$

and

$$
\left(\mathcal{I}_{t}^{\theta, v} \eta\right)(\omega)=\frac{1}{v^{\theta} \Gamma(\theta)} \int_{\omega}^{t} \exp \left[\frac{v-1}{v}(\kappa-\omega)\right](\kappa-\omega)^{\theta-1} \eta(\kappa) d \kappa
$$

where $\left({ }_{s} \mathcal{I}^{\theta, v} \eta\right)(\omega)$ and $\left(\mathcal{I}_{t}^{\theta, v} \eta\right)(\omega)$ are called the left- and right-sided proportional fractional integrals of a function $\eta$ of order $\theta$, respectively.

Definition 2.5 ([40]) Consider an integrable function $\eta$ and a strictly increasing continuous function $\psi$ on $[s, t]$, and let $v \in(0,1]$. We define, for all $\theta \in \mathbb{C}, \operatorname{Re}(\theta) \geq 0$,

$$
\begin{aligned}
\left({ }_{s}^{\psi} D^{\theta, v} \eta\right)(\omega)= & { }^{\psi} D_{s}^{m, v \psi} \mathcal{I}^{m-\theta, v} \eta(\omega) \\
= & \frac{{ }^{\psi} D_{\omega}^{m, v}}{v^{m-\theta} \Gamma(m-\theta)} \int_{s}^{\omega} \exp \left[\frac{v-1}{v}(\psi(\omega)-\psi(\kappa))\right] \\
& \times(\psi(\omega)-\psi(\kappa))^{m-\theta-1} \psi^{\prime}(\kappa) \eta(\kappa) d \kappa
\end{aligned}
$$

and

$$
\begin{aligned}
\left({ }^{\psi} D_{t}^{\theta, v} \eta\right)(\omega)= & { }_{\gamma}^{\psi} D^{m, v \psi} \mathcal{I}_{t}^{m-\theta, v} \eta(\omega) \\
= & \frac{{ }_{\gamma}^{\psi} D_{\omega}^{m, v}}{v^{m-\theta} \Gamma(m-\theta)} \int_{\omega}^{t} \exp \left[\frac{v-1}{v}(\psi(\kappa)-\psi(\omega))\right] \\
& \times(\psi(\kappa)-\psi(\omega))^{m-\theta-1} \psi^{\prime}(\kappa) \eta(\kappa) d \kappa,
\end{aligned}
$$


where

$$
{ }^{\psi} D^{m, v}=\underbrace{\psi D^{v \psi} D^{v} \ldots{ }^{\psi} D^{v}}_{m \text {-times }}, \quad m=[\operatorname{Re}(\theta)]+1,
$$

and

$$
\left({ }_{\gamma}^{\psi} D^{v} \eta\right)(\omega)=(1-v) \eta(\omega)-v \frac{\eta^{\prime}(\omega)}{\psi^{\prime}(\omega)}, \quad{ }_{\gamma}^{\psi} D^{m, v}=\underbrace{{ }_{\gamma}^{\psi} D_{\gamma}^{v \psi} D^{v} \ldots{ }_{\gamma}^{\psi} D^{v}}_{m \text {-times }} ;
$$

$\left({ }_{s}^{\psi} D^{\theta, v} \eta\right)(\omega)$ and $\left({ }^{\psi} D_{t}^{\theta, v} \eta\right)(\omega)$ are called respectively the left- and right-sided proportional fractional derivatives of a function $\eta$ with respect to the function $\psi$ of order $\theta$.

Definition 2.6 ([40]) Consider an integrable function $\eta$ and a strictly increasing continuous function $\psi$ on $[s, t]$, and let $v \in(0,1]$. We define, for all $\theta \in \mathbb{C}, \operatorname{Re}(\theta) \geq 0$,

$$
\begin{aligned}
\left({ }_{s}^{\psi} \mathcal{I}^{\theta, v} \eta\right)(\omega)= & \frac{1}{v^{\theta} \Gamma(\theta)} \int_{s}^{\omega} \exp \left[\frac{v-1}{v}(\psi(\omega)-\psi(\kappa))\right] \\
& \times(\psi(\omega)-\psi(\kappa))^{\theta-1} \psi^{\prime}(\kappa) \eta(\kappa) d \kappa
\end{aligned}
$$

and

$$
\begin{aligned}
\left({ }^{\psi} \mathcal{I}_{t}^{\theta, v} \eta\right)(\omega)= & \frac{1}{v^{\theta} \Gamma(\theta)} \int_{\omega}^{t} \exp \left[\frac{v-1}{v}(\psi(\kappa)-\psi(\omega))\right] \\
& \times(\psi(\kappa)-\psi(\omega))^{\theta-1} \psi^{\prime}(\kappa) \eta(\kappa) d \kappa,
\end{aligned}
$$

where $\left.{ }_{s}^{\psi} \mathcal{I}^{\theta, v} \eta\right)(\omega)$ and $\left({ }^{\psi} \mathcal{I}_{t}^{\theta, v} \eta\right)(\omega)$ are called respectively the left- and right-sided proportional fractional integrals of a function $\eta$ with respect to the function $\psi$ of order $\theta$.

Lemma 2.1 ([40]) Let $\psi$ be a continuous function on $\omega \geq$ s. If $v \in(0,1]$ and $\operatorname{Re}(\theta), \operatorname{Re}(\mu)>$ 0 , then we have

$$
\begin{aligned}
& { }_{s}^{\psi} \mathcal{I}^{\theta, v}\left({ }_{s}^{\psi} \mathcal{I}^{\mu, v} \eta\right)(\omega)={ }_{s}^{\psi} \mathcal{I}^{\mu, v}\left({ }_{s}^{\psi} \mathcal{I}^{\theta, v} \eta\right)(\omega)=\left({ }_{s}^{\psi} \mathcal{I}^{\theta+\mu, v} \eta\right)(\omega), \\
& { }^{\psi} \mathcal{I}_{t}^{\theta, v}\left({ }^{\psi} \mathcal{I}_{t}^{\mu, v} \eta\right)(\omega)={ }^{\psi} \mathcal{I}_{t}^{\mu, v}\left({ }^{\psi} \mathcal{I}_{t}^{\theta, v} \eta\right)(\omega)=\left({ }^{\psi} \mathcal{I}_{t}^{\theta+\mu, v} \eta\right)(\omega) .
\end{aligned}
$$

Lemma $2.2([40])$ Let $\psi$ be an integrable functions defined on $[s, \omega]$ or for $\omega>s$. If $0 \leq m<$ $[\operatorname{Re}(\theta)]+1$, then we have

$$
\begin{aligned}
& { }^{\psi} D^{m, v}\left({ }_{s}^{\psi} \mathcal{I}^{\theta, v} \eta\right)(\omega)=\left({ }_{s}^{\psi} \mathcal{I}^{\theta-m, v} \eta\right)(\omega), \\
& { }_{\gamma}^{\psi} D^{m, v}\left({ }^{\psi} \mathcal{I}_{t}^{\theta, v} \eta\right)(\omega)=\left({ }^{\psi} \mathcal{I}_{t}^{\theta-m, v} \eta\right)(\omega) .
\end{aligned}
$$

This paper is concerned with the use of left-sided $\psi$-proportional fractional integrals (2.11) to obtain the main results. 


\section{Reverse Minkowski's inequalities for fractional integral}

This part is concerned with our main results on the generalization and development of Minkowski's and related inequalities with the help of proportional fractional integral operator with respect to another strictly increasing continuous function $\psi$.

First, in order to state our results, we compute the left-sided $\psi$-proportional fractional integral for any constant as follows:

Let $v \in(0,1], \theta \in \mathbb{C}, \operatorname{Re}(\theta) \geq 0$, and $\psi$ be a strictly increasing continuous function. Then for any constant $k$, we have

$$
\left({ }_{s}^{\psi} \mathcal{I}^{\theta, v} k\right)(\omega)=\frac{(\psi(\omega)-\psi(s))^{\theta}}{v^{\theta} \Gamma(\theta+1)} k
$$

Theorem 3.1 Consider positive functions $\eta$, ऽ on $[0, \infty)$ with ${ }_{s}^{\psi} \mathcal{I}^{\theta, v} \eta^{z}(\omega),{ }_{s}^{\psi} \mathcal{I}^{\theta, v} \varsigma^{z}(\omega)<\infty$, $\forall \omega \in[0, \infty)$ and for all $v \in(0,1], \theta \in \mathbb{C}, \operatorname{Re}(\theta) \geq 0, z \geq 1$. Let $\psi$ be a strictly increasing continuous function. Assume that $\mathrm{€}, \varphi$ are two positive functions such that $0<\mathrm{€}(\varepsilon) \leq \frac{\eta(\kappa)}{\zeta(\kappa)} \leq$ $\varphi(\varepsilon), \kappa, \varepsilon \in[s, \omega]$. Then we have

$$
\begin{aligned}
{\left[{ }_{s}^{\psi} \mathcal{I}^{\theta, v} \eta^{z}(\omega)\right]^{\frac{1}{z}}+\left[{ }_{s}^{\psi} \mathcal{I}^{\theta, v} S^{z}(\omega)\right]^{\frac{1}{z}} } \\
\leq \frac{v^{\theta} \Gamma(\theta+1)}{(\psi(\omega)-\psi(s))^{\theta}}\left\{{ }_{s}^{\psi} \mathcal{I}^{\theta, v}\left(\frac{\varphi(\omega)}{\varphi(\omega)+1}\right)+{ }_{s}^{\psi} \mathcal{I}^{\theta, v}\left(\frac{1}{\mathrm{七}(\omega)+1}\right)\right\} \\
\quad \times\left({ }_{s}^{\psi} \mathcal{I}^{\theta, v}(\eta+\varsigma)^{z}(\omega)\right)^{\frac{1}{z}}
\end{aligned}
$$

Proof Due to the assumption $\frac{\eta(\kappa)}{\zeta(\varepsilon)} \leq \varphi(\varepsilon) ; \kappa, \varepsilon \in[s, \omega]$, we can write

$$
[\varphi(\varepsilon)+1]^{z} \eta^{z}(\kappa) \leq \varphi^{z}(\varepsilon)(\eta+\varsigma)^{z}(\kappa)
$$

and from the assumption $€(\varepsilon) \leq \frac{\eta(\kappa)}{\zeta(\varepsilon)} ; \kappa, \varepsilon \in[s, \omega]$, we have

$$
\left(1+\frac{1}{\mathrm{七}(\varepsilon)}\right)^{z} \varsigma^{z}(\kappa) \leq\left(\frac{1}{\mathrm{七}(\varepsilon)}\right)^{z}(\eta+\varsigma)^{z}(\kappa)
$$

Multiplying both sides of (3.3) and (3.4) by $\frac{1}{v^{\theta} \Gamma(\theta)} \times \frac{\exp \left[\frac{v-1}{v}(\psi(\omega)-\psi(\kappa))\right]}{(\psi(\omega)-\psi(\kappa))^{1-\theta}} \psi^{\prime}(\kappa), \kappa<\omega$, then integrating the results with respect to $\kappa$ over $(s, \omega)$, we respectively get

$$
\begin{aligned}
& \frac{[\varphi(\varepsilon)+1]^{z}}{v^{\theta} \Gamma(\theta)} \int_{s}^{\omega} \frac{\exp \left[\frac{v-1}{v}(\psi(\omega)-\psi(\kappa))\right]}{(\psi(\omega)-\psi(\kappa))^{1-\theta}} \psi^{\prime}(\kappa) \eta^{z}(\kappa) d \kappa \\
& \quad \leq \frac{\varphi^{z}(\varepsilon)}{v^{\theta} \Gamma(\theta)} \int_{s}^{\omega} \frac{\exp \left[\frac{v-1}{v}(\psi(\omega)-\psi(\kappa))\right]}{(\psi(\omega)-\psi(\kappa))^{1-\theta}} \psi^{\prime}(\kappa)(\eta+\varsigma)^{z}(\kappa) d \kappa
\end{aligned}
$$

and

$$
\begin{aligned}
& \left(1+\frac{1}{\mathrm{Ł}(\varepsilon)}\right)^{z} \frac{1}{v^{\theta} \Gamma(\theta)} \int_{s}^{\omega} \frac{\exp \left[\frac{v-1}{v}(\psi(\omega)-\psi(\kappa))\right]}{(\psi(\omega)-\psi(\kappa))^{1-\theta}} \psi^{\prime}(\kappa) \varsigma^{z}(\kappa) d \kappa \\
& \leq\left(\frac{1}{€(\varepsilon)}\right)^{z} \frac{1}{v^{\theta} \Gamma(\theta)} \int_{s}^{\omega} \frac{\exp \left[\frac{v-1}{v}(\psi(\omega)-\psi(\kappa))\right]}{(\psi(\omega)-\psi(\kappa))^{1-\theta}} \psi^{\prime}(\kappa)(\eta+\varsigma)^{z}(\kappa) d \kappa .
\end{aligned}
$$


So we have

$$
[\varphi(\varepsilon)+1]_{s}^{z \psi} \mathcal{I}^{\theta, v} \eta^{z}(\omega) \leq \varphi^{z}(\varepsilon)_{s}^{\psi} \mathcal{I}^{\theta, v}(\eta+\varsigma)^{z}(\omega)
$$

and

$$
\left(1+\frac{1}{\mathrm{E}(\varepsilon)}\right)_{s}^{z} \mathcal{I}^{\theta, v} \varsigma^{z}(\omega) \leq\left(\frac{1}{\mathrm{\complement}(\varepsilon)}\right)_{s}^{z} \mathcal{I}^{\theta, v}(\eta+\varsigma)^{z}(\omega),
$$

which can be written as

$$
\left({ }_{s}^{\psi} \mathcal{I}^{\theta, v} \eta^{z}(\omega)\right)^{\frac{1}{z}} \leq \frac{\varphi(\varepsilon)}{\varphi(\varepsilon)+1}\left({ }_{s}^{\psi} \mathcal{I}^{\theta, v}(\eta+\varsigma)^{z}(\omega)\right)^{\frac{1}{z}}
$$

and

$$
\left({ }_{s}^{\psi} \mathcal{I}^{\theta, v} \varsigma^{z}(\omega)\right)^{\frac{1}{z}} \leq \frac{1}{€(\varepsilon)+1}\left({ }_{s}^{\psi} \mathcal{I}^{\theta, v}(\eta+\varsigma)^{z}(\omega)\right)^{\frac{1}{z}} .
$$

Analogously, multiplying both sides of (3.7) and (3.8) by $\frac{1}{v^{\theta} \Gamma(\theta)} \times \frac{\exp \left[\frac{v-1}{v}(\psi(\omega)-\psi(\varepsilon))\right]}{(\psi(\omega)-\psi(\varepsilon))^{1-\theta}} \psi^{\prime}(\varepsilon)$, $\varepsilon<\omega$, then integrating the results with respect to $\varepsilon$ over $(s, \omega)$, we respectively obtain

$$
\begin{aligned}
& \left({ }_{s}^{\psi} \mathcal{I}^{\theta, v} \eta^{z}(\omega)\right)^{\frac{1}{z}} \frac{1}{v^{\theta} \Gamma(\theta)} \int_{s}^{\omega} \frac{\exp \left[\frac{v-1}{v}(\psi(\omega)-\psi(\varepsilon))\right]}{(\psi(\omega)-\psi(\varepsilon))^{1-\theta}} \psi^{\prime}(\varepsilon) d \varepsilon \\
& \quad \leq\left({ }_{s}^{\psi} \mathcal{I}^{\theta, v}(\eta+\varsigma)^{z}(\omega)\right)^{\frac{1}{z}} \frac{1}{v^{\theta} \Gamma(\theta)} \int_{s}^{\omega} \frac{\exp \left[\frac{v-1}{v}(\psi(\omega)-\psi(\varepsilon))\right]}{(\psi(\omega)-\psi(\varepsilon))^{1-\theta}} \frac{\psi^{\prime}(\varepsilon) \varphi(\varepsilon)}{\varphi(\varepsilon)+1} d \varepsilon
\end{aligned}
$$

and

$$
\begin{aligned}
& \left({ }_{s}^{\psi} \mathcal{I}^{\theta, v} \varsigma^{z}(\omega)\right)^{\frac{1}{z}} \frac{1}{v^{\theta} \Gamma(\theta)} \int_{s}^{\omega} \frac{\exp \left[\frac{v-1}{v}(\psi(\omega)-\psi(\varepsilon))\right]}{(\psi(\omega)-\psi(\varepsilon))^{1-\theta}} \psi^{\prime}(\varepsilon) d \varepsilon \\
& \quad \leq\left({ }_{s}^{\psi} \mathcal{I}^{\theta, v}(\eta+\varsigma)^{z}(\omega)\right)^{\frac{1}{z}} \frac{1}{v^{\theta} \Gamma(\theta)} \int_{s}^{\omega} \frac{\exp \left[\frac{v-1}{v}(\psi(\omega)-\psi(\varepsilon))\right]}{(\psi(\omega)-\psi(\varepsilon))^{1-\theta}} \frac{\psi^{\prime}(\varepsilon)}{\mathrm{E}(\varepsilon)+1} d \varepsilon
\end{aligned}
$$

which yields

$$
\frac{(\psi(\omega)-\psi(s))^{\theta}}{v^{\theta} \Gamma(\theta+1)}\left({ }_{s}^{\psi} \mathcal{I}^{\theta, v} \eta^{z}(\omega)\right)^{\frac{1}{z}} \leq{ }_{s}^{\psi} \mathcal{I}^{\theta, v}\left(\frac{\varphi(\omega)}{\varphi(\omega)+1}\right)\left({ }_{s}^{\psi} \mathcal{I}^{\theta, v}(\eta+\varsigma)^{z}(\omega)\right)^{\frac{1}{z}}
$$

and

$$
\frac{(\psi(\omega)-\psi(s))^{\theta}}{v^{\theta} \Gamma(\theta+1)}\left({ }_{s}^{\psi} \mathcal{I}^{\theta, v} \varsigma^{z}(\omega)\right)^{\frac{1}{z}} \leq{ }_{s}^{\psi} \mathcal{I}^{\theta, v}\left(\frac{1}{€(\omega)+1}\right)\left({ }_{s}^{\psi} \mathcal{I}^{\theta, v}(\eta+\varsigma)^{z}(\omega)\right)^{\frac{1}{z}} .
$$

Consequently, the addition of (3.9) and (3.10) completes the proof.

As a special case, the following corollary is a two-parameter version of Theorem 3.1.

Corollary 3.1 Consider positive functions $\eta, \varsigma$ on $[0, \infty)$ with ${ }_{s}^{\psi} \mathcal{I}^{\theta, v} \eta^{z}(\omega),{ }_{s}^{\psi} \mathcal{I}^{\theta, v} \varsigma^{z}(\omega)<\infty$, $\forall \omega \in[0, \infty)$ and for all $v \in(0,1], \theta, \mu \in \mathbb{C}, \operatorname{Re}(\theta), \operatorname{Re}(\mu) \geq 0, z \geq 1$. Let $\psi$ be a strictly 
increasing continuous function. Assume that $\mathrm{t}, \varphi$ are two positive functions such that $0<$ $\mathrm{\iota}(\varepsilon) \leq \frac{\eta(\kappa)}{\zeta(\kappa)} \leq \varphi(\varepsilon), \kappa, \varepsilon \in[s, \omega]$. Then we have

$$
\begin{aligned}
& {\left[{ }_{s}^{\psi} \mathcal{I}^{\theta, v} \eta^{z}(\omega)\right]^{\frac{1}{z}}+\left[{ }_{s}^{\psi} \mathcal{I}^{\theta, v} \varsigma^{z}(\omega)\right]^{\frac{1}{z}}} \\
& \leq \frac{v^{\mu} \Gamma(\mu+1)}{(\psi(\omega)-\psi(s))^{\mu}}\left\{{ }_{s}^{\psi} \mathcal{I}^{\mu, v}\left(\frac{\varphi(\omega)}{\varphi(\omega)+1}\right)+{ }_{s}^{\psi} \mathcal{I}^{\mu, v}\left(\frac{1}{\mathrm{E}(\omega)+1}\right)\right\} \\
& \quad \times\left({ }_{s}^{\psi} \mathcal{I}^{\theta, v}(\eta+\varsigma)^{z}(\omega)\right)^{\frac{1}{z}} .
\end{aligned}
$$

Proof We can easily prove (3.11) by multiplying both sides of (3.7) and (3.8) by

$$
\frac{1}{v^{\mu} \Gamma(\mu)} \times \frac{\exp \left[\frac{v-1}{v}(\psi(\omega)-\psi(\varepsilon))\right]}{(\psi(\omega)-\psi(\varepsilon))^{1-\mu}} \psi^{\prime}(\varepsilon), \quad \varepsilon<\omega
$$

then integrating the results with respect to $\varepsilon$ over $(s, \omega)$. We can conclude the proof using the same argument as that in the proof of Theorem 3.1.

The next theorem is as follows:

Theorem 3.2 Consider positive functions $\eta$, ऽ on $[0, \infty)$ with ${ }_{s}^{\psi} \mathcal{I}^{\theta, v} \eta^{z}(\omega),{ }_{s}^{\psi} \mathcal{I}^{\theta, v} \varsigma^{z}(\omega)<\infty$, $\forall \omega \in[0, \infty)$ and for all $v \in(0,1], \theta \in \mathbb{C}, \operatorname{Re}(\theta) \geq 0, z \geq 1$. Let $\psi$ be a strictly increasing

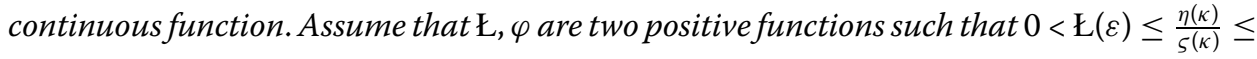
$\varphi(\varepsilon), \kappa, \varepsilon \in[s, \omega]$. Then we have

$$
\begin{aligned}
\left({ }_{s}^{\psi} \mathcal{I}^{\theta, v} \eta^{z}(\omega)\right)^{\frac{2}{z}}+\left({ }_{s}^{\psi} \mathcal{I}^{\theta, v} \varsigma^{z}(\omega)\right)^{\frac{2}{z}} \\
\geq \\
\geq\left\{\left[\frac{v^{2 \theta} \Gamma^{2}(\theta+1)}{(\psi(\omega)-\psi(s))^{2 \theta}}\right]{ }_{s}^{\psi} \mathcal{I}^{\theta, v}\left(\frac{\varphi(\omega)+1}{\varphi(\omega)}\right){ }_{s}^{\psi} \mathcal{I}^{\theta, v}(\mathrm{E}(\omega)+1)-2\right\} \\
\quad \times\left({ }_{s}^{\psi} \mathcal{I}^{\theta, v} \eta^{z}(\omega)\right)^{\frac{1}{z}}\left({ }_{s}^{\psi} \mathcal{I}^{\theta, v} \varsigma^{z}(\omega)\right)^{\frac{1}{z}} .
\end{aligned}
$$

Proof Here, to get started, we reformulate inequalities (3.7) and (3.8), respectively, as

$$
\frac{\varphi(\varepsilon)+1}{\varphi(\varepsilon)}\left({ }_{s}^{\psi} \mathcal{I}^{\theta, v} \eta^{z}(\omega)\right)^{\frac{1}{z}} \leq\left({ }_{s}^{\psi} \mathcal{I}^{\theta, v}(\eta+\varsigma)^{z}(\omega)\right)^{\frac{1}{z}}
$$

and

$$
(\mathrm{E}(\varepsilon)+1)\left({ }_{s}^{\psi} \mathcal{I}^{\theta, v} \varsigma^{z}(\omega)\right)^{\frac{1}{z}} \leq\left({ }_{s}^{\psi} \mathcal{I}^{\theta, v}(\eta+\varsigma)^{z}(\omega)\right)^{\frac{1}{z}} .
$$

Multiplying both sides of (3.13) and (3.14) by $\frac{1}{v^{\theta} \Gamma(\theta)} \times \frac{\exp \left[\frac{v-1}{v}(\psi(\omega)-\psi(\varepsilon))\right]}{(\psi(\omega)-\psi(\varepsilon))^{1-\theta}} \psi^{\prime}(\varepsilon), \varepsilon<\omega$, then integrating the results with respect to $\varepsilon$ over $(s, \omega)$, we respectively obtain

$$
\left[\frac{v^{\theta} \Gamma(\theta+1)}{(\psi(\omega)-\psi(s))^{\theta}}\right]{ }_{s}^{\psi} \mathcal{I}^{\theta, v}\left(\frac{\varphi(\omega)+1}{\varphi(\omega)}\right)\left({ }_{s}^{\psi} \mathcal{I}^{\theta, v} \eta^{z}(\omega)\right)^{\frac{1}{z}} \leq\left({ }_{s}^{\psi} \mathcal{I}^{\theta, v}(\eta+\varsigma)^{z}(\omega)\right)^{\frac{1}{z}}
$$

and

$$
\left[\frac{v^{\theta} \Gamma(\theta+1)}{(\psi(\omega)-\psi(s))^{\theta}}\right]_{s}^{\psi} \mathcal{I}^{\theta, v}(\mathrm{E}(\omega)+1)\left({ }_{s}^{\psi} \mathcal{I}^{\theta, v} \varsigma^{z}(\omega)\right)^{\frac{1}{z}} \leq\left({ }_{s}^{\psi} \mathcal{I}^{\theta, v}(\eta+\varsigma)^{z}(\omega)\right)^{\frac{1}{z}} .
$$


Now, taking the product of corresponding parts of inequalities (3.15) and (3.16), we obtain

$$
\begin{aligned}
& {\left[\frac{v^{2 \theta} \Gamma^{2}(\theta+1)}{(\psi(\omega)-\psi(s))^{2 \theta}}\right]_{s}^{\psi} \mathcal{I}^{\theta, v}\left(\frac{\varphi(\omega)+1}{\varphi(\omega)}\right){ }_{s}^{\psi} \mathcal{I}^{\theta, v}(\mathrm{E}(\omega)+1)\left({ }_{s}^{\psi} \mathcal{I}^{\theta, v} \eta^{z}(\omega)\right)^{\frac{1}{z}}\left({ }_{s}^{\psi} \mathcal{I}^{\theta, v} \varsigma^{z}(\omega)\right)^{\frac{1}{z}}} \\
& \leq\left(\left(\left(_{s}^{\psi} \mathcal{I}^{\theta, v}(\eta+\varsigma)^{z}(\omega)\right)^{\frac{1}{z}}\right)^{2} .\right.
\end{aligned}
$$

On the right-hand side of (3.17), we apply Minkowski inequality to obtain

$$
\left(\left({ }_{s}^{\psi} \mathcal{I}^{\theta, v}(\eta+\varsigma)^{z}(\omega)\right)^{\frac{1}{z}}\right)^{2} \leq\left(\left({ }_{s}^{\psi} \mathcal{I}^{\theta, v} \eta^{z}(\omega)\right)^{\frac{1}{z}}+\left({ }_{s}^{\psi} \mathcal{I}^{\theta, v} \varsigma^{z}(\omega)\right)^{\frac{1}{z}}\right)^{2}
$$

which yields

$$
\begin{aligned}
\left(\left({ }_{s}^{\psi} \mathcal{I}^{\theta, v}(\eta+\varsigma)^{z}(\omega)\right)^{\frac{1}{z}}\right)^{2} \leq & \left({ }_{s}^{\psi} \mathcal{I}^{\theta, v} \eta^{z}(\omega)\right)^{\frac{2}{z}}+\left({ }_{s}^{\psi} \mathcal{I}^{\theta, v} \varsigma^{z}(\omega)\right)^{\frac{2}{z}} \\
& +2\left({ }_{s}^{\psi} \mathcal{I}^{\theta, v} \eta^{z}(\omega)\right)^{\frac{1}{z}}\left({ }_{s}^{\psi} \mathcal{I}^{\theta, v} \varsigma^{z}(\omega)\right)^{\frac{1}{z}}
\end{aligned}
$$

By matching inequality (3.17) with (3.18), we obtain the required inequality (3.12).

\section{Remark 3.1}

(i) In Theorems 3.1 and 3.2, putting $v=1$ and $\psi(\omega)=\omega(\forall \omega \in[s, t], s=0)$, we get Theorems 3.1 and 3.3, respectively, proved in [37].

(ii) In Theorems 3.1 and 3.2, putting $s=0, v=1, \mathrm{t}(\omega)=q$, and $\varphi(\omega)=Q, \psi(\omega)=\omega$ $(\forall \omega \in[s, t]$ where $q, Q$ are constants), we get Theorems 2.1 and 2.3, respectively, introduced by Dahmani [19].

(iii) Applying Theorems 3.1 and 3.2 with $\theta=1, v=1, \psi(\omega)=\omega, \mathrm{Ł}(\omega)=q$, and $\varphi(\omega)=Q$ on $[s, w]$, we obtain Theorem 1.2 introduced by Bougoffa [18] and Theorem 2.2 proved by Set et al. [20], respectively.

\section{Other related $\psi$-proportional fractional integral inequalities}

This section is devoted to deducing some fractional integral inequalities in the case of functional boundaries, which are close to the fractional Minkowski integral inequalities.

Theorem 4.1 Consider positive functions $\eta$, ऽ on $[0, \infty)$ with ${ }_{s}^{\psi} \mathcal{I}^{\theta, v} \eta^{z}(\omega),{ }_{s}^{\psi} \mathcal{I}^{\theta, v} \varsigma^{z}(\omega)<\infty$, $\forall \omega \in[0, \infty)$ and for all $v \in(0,1], \theta \in \mathbb{C}, \operatorname{Re}(\theta) \geq 0, z, l \geq 1, z, l \in \mathbb{R}_{+}^{*}$ where $\frac{1}{z}+\frac{1}{l}=1$. Let $\psi$ be a strictly increasing continuous function. Assume that,$\varphi$ are two positive functions such that $0<\mathrm{E}(\varepsilon) \leq \frac{\eta(\kappa)}{5(\kappa)} \leq \varphi(\varepsilon), \kappa, \varepsilon \in[s, \omega]$. Then we have

$$
\begin{aligned}
{ }_{s}^{\psi} \mathcal{I}^{\theta, v}(\eta \zeta)(\omega) \leq & {\left[\frac{2^{\lambda-1} v^{\theta} \Gamma(\theta+1)}{z(\psi(\omega)-\psi(s))^{\theta}}\right]{ }_{s}^{\psi} \mathcal{I}^{\theta, v}\left(\frac{\varphi^{z}(\omega)}{[\varphi(\omega)+1]^{z}}\right){ }_{s}^{\psi} \mathcal{I}^{\theta, v}\left[\eta^{z}(\omega)+\varsigma^{z}(\omega)\right] } \\
& +\left[\frac{2^{\lambda-1} v^{\theta} \Gamma(\theta+1)}{l(\psi(\omega)-\psi(s))^{\theta}}\right]{ }_{s}^{\psi} \mathcal{I}^{\theta, v}\left(\frac{1}{(\mathrm{E}(\omega)+1)^{l}}\right){ }_{s}^{\psi} \mathcal{I}^{\theta, v}\left[\eta^{l}(\omega)+\varsigma^{l}(\omega)\right] .
\end{aligned}
$$

Proof Due to the assumption $\frac{\eta(\kappa)}{\zeta(\varepsilon)} \leq \varphi(\varepsilon) ; \kappa, \varepsilon \in[s, \omega]$, we can write

$$
[\varphi(\varepsilon)+1]^{z} \eta^{z}(\kappa) \leq \varphi^{z}(\varepsilon)(\eta+\varsigma)^{z}(\kappa)
$$


and from the assumption $€(\varepsilon) \leq \frac{\eta(\kappa)}{\varsigma^{(\varepsilon)}} ; \kappa, \varepsilon \in[s, \omega]$, we obtain

$$
(\mathrm{E}(\varepsilon)+1)^{l} \varsigma^{l}(\kappa) \leq(\eta+\varsigma)^{l}(\kappa) .
$$

Multiplying both sides of (4.2) and (4.3) by $\frac{1}{v^{\theta} \Gamma(\theta)} \times \frac{\exp \left[\frac{v-1}{v}(\psi(\omega)-\psi(\kappa))\right]}{(\psi(\omega)-\psi(\kappa))^{1-\theta}} \psi^{\prime}(\kappa), \kappa<\omega$, then integrating the results with respect to $\kappa$ over $(s, \omega)$, we respectively get

$$
{ }_{s}^{\psi} \mathcal{I}^{\theta, v} \eta^{z}(\omega) \leq\left(\frac{\varphi^{z}(\varepsilon)}{[\varphi(\varepsilon)+1]^{z}}\right){ }_{s}^{\psi} \mathcal{I}^{\theta, v}(\eta+\varsigma)^{z}(\omega)
$$

and

$$
{ }_{s}^{\psi} \mathcal{I}^{\theta, v} \varsigma^{l}(\omega) \leq\left(\frac{1}{(\mathrm{E}(\varepsilon)+1)^{l}}\right){ }_{s}^{\psi} \mathcal{I}^{\theta, v}(\eta+\varsigma)^{l}(\omega)
$$

Again, multiplying both sides of (4.4) and (4.5) by $\frac{1}{v^{\theta} \Gamma(\theta)} \times \frac{\exp \left[\frac{\nu-1}{v}(\psi(\omega)-\psi(\varepsilon))\right]}{(\psi(\omega)-\psi(\varepsilon))^{1-\theta}} \psi^{\prime}(\varepsilon), \varepsilon<\omega$, then integrating the results with respect to $\varepsilon$ over $(s, \omega)$, we respectively obtain

$$
{ }_{s}^{\psi} \mathcal{I}^{\theta, v} \eta^{z}(\omega) \leq\left[\frac{v^{\theta} \Gamma(\theta+1)}{(\psi(\omega)-\psi(s))^{\theta}}\right]{ }_{s}^{\psi} \mathcal{I}^{\theta, v}\left(\frac{\varphi^{z}(\omega)}{[\varphi(\omega)+1]^{z}}\right){ }_{s}^{\psi} \mathcal{I}^{\theta, v}(\eta+\varsigma)^{z}(\omega)
$$

and

$$
{ }_{s}^{\psi} \mathcal{I}^{\theta, v} \varsigma^{l}(\omega) \leq\left[\frac{v^{\theta} \Gamma(\theta+1)}{(\psi(\omega)-\psi(s))^{\theta}}\right]_{s}^{\psi} \mathcal{I}^{\theta, v}\left(\frac{1}{(\mathrm{E}(\omega)+1)^{l}}\right){ }_{s}^{\psi} \mathcal{I}^{\theta, v}(\eta+\varsigma)^{l}(\omega)
$$

Now, considering Young's inequality [10],

$$
\eta(\kappa) \varsigma(\kappa) \leq \frac{\eta^{z}(\kappa)}{z}+\frac{\varsigma^{l}(\kappa)}{l} .
$$

Multiplying both sides of (4.8) by $\frac{1}{v^{\theta} \Gamma(\theta)} \times \frac{\exp \left[\frac{v-1}{v}(\psi(\omega)-\psi(\kappa))\right]}{(\psi(\omega)-\psi(\kappa))^{1-\theta}} \psi^{\prime}(\kappa), \kappa<\omega$, then integrating the result with respect to $\kappa$ over $(s, \omega)$, we get

$$
{ }_{s}^{\psi} \mathcal{I}^{\theta, v}(\eta \varsigma)(\omega) \leq \frac{1}{z^{\psi}} \mathcal{I}^{\theta, v} \eta^{z}(\omega)+\frac{1}{l}{ }^{\psi} \mathcal{I}^{\theta, v} \varsigma^{l}(\omega) .
$$

Inequalities (4.6), (4.7), and (4.9) lead to

$$
\begin{aligned}
{ }_{s}^{\psi} \mathcal{I}^{\theta, v}(\eta \varsigma)(\omega) \leq & \frac{1}{z^{s}} \mathcal{I}^{\theta, v} \eta^{z}(\omega)+\frac{1}{l} \psi \mathcal{I}^{\theta, v} \varsigma^{l}(\omega) \\
\leq & {\left[\frac{v^{\theta} \Gamma(\theta+1)}{z(\psi(\omega)-\psi(s))^{\theta}}\right]{ }_{s}^{\psi} \mathcal{I}^{\theta, v}\left(\frac{\varphi^{z}(\omega)}{[\varphi(\omega)+1]^{z}}\right){ }_{s} \mathcal{I}^{\theta, v}(\eta+\varsigma)^{z}(\omega) } \\
& +\left[\frac{v^{\theta} \Gamma(\theta+1)}{l(\psi(\omega)-\psi(s))^{\theta}}\right]{ }_{s}^{\psi} \mathcal{I}^{\theta, v}\left(\frac{1}{(\mathrm{七}(\omega)+1)^{l}}\right){ }_{s}^{\psi} \mathcal{I}^{\theta, v}(\eta+\varsigma)^{l}(\omega) .
\end{aligned}
$$

Now, due to the inequality $(\Xi+\Omega)^{\lambda} \leq 2^{\lambda-1}\left(\Xi^{\lambda}+\Omega^{\lambda}\right), \lambda>1, \Xi, \Omega \geq 0$, we can write

$$
{ }_{s}^{\psi} \mathcal{I}^{\theta, v}(\eta+\varsigma)^{z}(\omega) \leq 2_{s}^{\lambda-1 \psi} \mathcal{I}^{\theta, v}\left[\eta^{z}(\omega)+\varsigma^{z}(\omega)\right]
$$


and

$$
{ }_{s}^{\psi} \mathcal{I}^{\theta, v}(\eta+\varsigma)^{l}(\omega) \leq 2_{s}^{\lambda-1 \psi} \mathcal{I}^{\theta, v}\left[\eta^{l}(\omega)+\varsigma^{l}(\omega)\right]
$$

By substituting inequalities (4.11) and (4.12) into (4.10), we obtain inequality (4.1), which ends the proof.

Theorem 4.2 Consider positive functions $\eta, \varsigma$ on $[0, \infty)$ with ${ }_{s}^{\psi} \mathcal{I}^{\theta, v} \eta^{z}(\omega),{ }_{s}^{\psi} \mathcal{I}^{\theta, v} \varsigma^{z}(\omega)<\infty$, $\forall \omega \in[0, \infty)$ and for all $v \in(0,1], \theta \in \mathbb{C}, \operatorname{Re}(\theta) \geq 0, z \geq 1$. Let $\psi$ be a strictly increasing continuous function. Assume that $\mathrm{七}$, $\varphi$ are two positive functions such that $0<\Xi<\mathrm{\Xi}(\varepsilon) \leq$ $\frac{\eta(\kappa)}{\varsigma(\kappa)} \leq \varphi(\varepsilon), \kappa, \varepsilon \in[s, \omega]$. Then we have

$$
\begin{aligned}
& \frac{v^{\theta} \Gamma(\theta+1)}{(\psi(\omega)-\psi(s))^{\theta}}\left\{{ }_{s}^{\psi} \mathcal{I}^{\theta, v}\left(\frac{1}{(\varphi(\omega)-\Xi)}\right)+{ }_{s}^{\psi} \mathcal{I}^{\theta, v}\left(\frac{\mathrm{E}(\omega)}{\mathrm{\complement}(\omega)-\Xi}\right)\right\}\left[{ }_{s}^{\psi} \mathcal{I}^{\theta, v}(\eta(\omega)-\Xi \varsigma(\omega))^{z}\right]^{\frac{1}{z}} \\
& \leq\left({ }_{s}^{\psi} \mathcal{I}^{\theta, v} \varsigma^{z}(\omega)\right)^{\frac{1}{z}}+\left({ }_{s}^{\psi} \mathcal{I}^{\theta, v} \eta^{z}(\omega)\right)^{\frac{1}{z}} \\
& \leq \frac{v^{\theta} \Gamma(\theta+1)}{(\psi(\omega)-\psi(s))^{\theta}}\left\{{ }_{s}^{\psi} \mathcal{I}^{\theta, v}\left(\frac{1}{(\mathrm{~L}(\omega)-\Xi)}\right)+{ }_{s}^{\psi} \mathcal{I}^{\theta, v}\left(\frac{\varphi(\omega)}{\varphi(\omega)-\Xi}\right)\right\} \\
& \quad \times\left[{ }_{s}^{\psi} \mathcal{I}^{\theta, v}(\eta(\omega)-\Xi \varsigma(\omega))^{z}\right]^{\frac{1}{z}} .
\end{aligned}
$$

Proof Due to the assumption $0<\Xi<\mathrm{\iota}(\varepsilon) \leq \frac{\eta(\kappa)}{\zeta(\kappa)} \leq \varphi(\varepsilon)$, we can write

$$
€(\varepsilon) \leq \frac{\eta(\kappa)}{\varsigma(\kappa)} \leq \varphi(\varepsilon) \quad \Longrightarrow \quad 七(\varepsilon)-\Xi \leq \frac{\eta(\kappa)}{\varsigma(\kappa)}-\Xi \leq \varphi(\varepsilon)-\Xi
$$

So

$$
£(\varepsilon)-\Xi \leq \frac{\eta(\kappa)-\Xi \varsigma(\kappa)}{\varsigma(\kappa)} \leq \varphi(\varepsilon)-\Xi
$$

which leads to

$$
\frac{(\eta(\kappa)-\Xi \varsigma(\kappa))^{z}}{(\varphi(\varepsilon)-\Xi)^{z}} \leq \varsigma^{z}(\kappa) \leq \frac{(\eta(\kappa)-\Xi \varsigma(\kappa))^{z}}{(Ł(\varepsilon)-\Xi)^{z}}
$$

Also, we have

$$
€(\varepsilon) \leq \frac{\eta(\kappa)}{\varsigma(\kappa)} \leq \varphi(\varepsilon) \Longrightarrow \frac{1}{\Xi}-\frac{1}{£(\varepsilon)} \leq \frac{1}{\Xi}-\frac{\varsigma(\kappa)}{\eta(\kappa)} \leq \frac{1}{\Xi}-\frac{1}{\varphi(\varepsilon)},
$$

so

$$
\frac{€(\varepsilon)-\Xi}{€(\varepsilon)} \leq \frac{\eta(\kappa)-\Xi \varsigma(\kappa)}{\eta(\kappa)} \leq \frac{\varphi(\varepsilon)-\Xi}{\varphi(\varepsilon)}
$$

which leads to

$$
\left(\frac{\mathrm{七}(\varepsilon)}{\mathrm{七}(\varepsilon)-\Xi}\right)^{z}\left(\eta(\kappa)-\Xi_{\varsigma}(\kappa)\right)^{z} \leq \eta^{z}(\kappa) \leq\left(\frac{\varphi(\varepsilon)}{\varphi(\varepsilon)-\Xi}\right)^{z}\left(\eta(\kappa)-\Xi_{\varsigma}(\kappa)\right)^{z}
$$


Multiplying both sides of (4.14) and (4.15) by $\frac{1}{v^{\theta} \Gamma(\theta)} \times \frac{\exp \left[\frac{v-1}{v}(\psi(\omega)-\psi(\kappa))\right]}{(\psi(\omega)-\psi(\kappa))^{1-\theta}} \psi^{\prime}(\kappa), \kappa<\omega$, then integrating the results with respect to $\kappa$ over $(s, \omega)$, we respectively get

$$
\begin{aligned}
& \frac{1}{v^{\theta} \Gamma(\theta)(\varphi(\varepsilon)-\Xi)^{z}} \int_{s}^{\omega} \frac{\exp \left[\frac{v-1}{v}(\psi(\omega)-\psi(\kappa))\right]}{(\psi(\omega)-\psi(\kappa))^{1-\theta}}\left(\eta(\kappa)-\Xi_{\varsigma}(\kappa)\right)^{z} \psi^{\prime}(\kappa) d \kappa \\
& \quad \leq \frac{1}{v^{\theta} \Gamma(\theta)} \int_{s}^{\omega} \frac{\exp \left[\frac{v-1}{v}(\psi(\omega)-\psi(\kappa))\right]}{(\psi(\omega)-\psi(\kappa))^{1-\theta}} \varsigma^{z}(\kappa) \psi^{\prime}(\kappa) d \kappa \\
& \quad \leq \frac{1}{v^{\theta} \Gamma(\theta)(Ł(\varepsilon)-\Xi)^{z}} \int_{s}^{\omega} \frac{\exp \left[\frac{v-1}{v}(\psi(\omega)-\psi(\kappa))\right]}{(\psi(\omega)-\psi(\kappa))^{1-\theta}}\left(\eta(\kappa)-\Xi_{\zeta}(\kappa)\right)^{z} \psi^{\prime}(\kappa) d \kappa
\end{aligned}
$$

and

$$
\begin{aligned}
& \left(\frac{\mathrm{七}(\varepsilon)}{\mathrm{七}(\varepsilon)-\Xi}\right)^{z} \frac{1}{v^{\theta} \Gamma(\theta)} \int_{s}^{\omega} \frac{\exp \left[\frac{v-1}{v}(\psi(\omega)-\psi(\kappa))\right]}{(\psi(\omega)-\psi(\kappa))^{1-\theta}}\left(\eta(\kappa)-\Xi_{\zeta}(\kappa)\right)^{z} \psi^{\prime}(\kappa) d \kappa \\
& \quad \leq \frac{1}{v^{\theta} \Gamma(\theta)} \int_{s}^{\omega} \frac{\exp \left[\frac{v-1}{v}(\psi(\omega)-\psi(\kappa))\right]}{(\psi(\omega)-\psi(\kappa))^{1-\theta}} \eta^{z}(\kappa) \psi^{\prime}(\kappa) d \kappa \\
& \quad \leq\left(\frac{\varphi(\varepsilon)}{\varphi(\varepsilon)-\Xi}\right)^{z} \frac{1}{v^{\theta} \Gamma(\theta)} \int_{s}^{\omega} \frac{\exp \left[\frac{v-1}{v}(\psi(\omega)-\psi(\kappa))\right]}{(\psi(\omega)-\psi(\kappa))^{1-\theta}}\left(\eta(\kappa)-\Xi_{\zeta}(\kappa)\right)^{z} \psi^{\prime}(\kappa) d \kappa,
\end{aligned}
$$

from which it follows that

$$
\begin{aligned}
& \frac{1}{(\varphi(\varepsilon)-\Xi)^{z}}{ }^{\psi} \mathcal{I}^{\theta, v}\left(\eta(\omega)-\Xi_{\zeta}(\omega)\right)^{z} \\
& \quad \leq{ }_{s}^{\psi} \mathcal{I}^{\theta, v} \varsigma^{z}(\omega) \leq \frac{1}{(\mathrm{七}(\varepsilon)-\Xi)^{z}}{ }^{\psi} \mathcal{I}^{\theta, v}\left(\eta(\omega)-\Xi_{\zeta}(\omega)\right)^{z}
\end{aligned}
$$

and

$$
\begin{aligned}
& \left(\frac{\mathrm{\complement}(\varepsilon)}{\mathrm{七}(\varepsilon)-\Xi}\right)^{z}{ }_{s}^{\psi} \mathcal{I}^{\theta, v}\left(\eta(\omega)-\Xi_{\varsigma}(\omega)\right)^{z} \\
& \quad \leq{ }_{s}^{\psi} \mathcal{I}^{\theta, v} \eta^{z}(\omega) \leq\left(\frac{\varphi(\varepsilon)}{\varphi(\varepsilon)-\Xi}\right)_{s}^{z} \mathcal{I}^{\theta, v}\left(\eta(\omega)-\Xi_{\varsigma}(\omega)\right)^{z} .
\end{aligned}
$$

The above inequalities are respectively equivalent to

$$
\begin{aligned}
& \frac{1}{(\varphi(\varepsilon)-\Xi)}\left[{ }_{s}^{\psi} \mathcal{I}^{\theta, v}\left(\eta(\omega)-\Xi_{\zeta}(\omega)\right)^{z}\right]^{\frac{1}{z}} \\
& \quad \leq\left({ }_{s}^{\psi} \mathcal{I}^{\theta, v} \varsigma^{z}(\omega)\right)^{\frac{1}{z}} \leq \frac{1}{(\mathrm{\complement}(\varepsilon)-\Xi)}\left[{ }_{s}^{\psi} \mathcal{I}^{\theta, v}\left(\eta(\omega)-\Xi_{\zeta}(\omega)\right)^{z}\right]^{\frac{1}{z}}
\end{aligned}
$$

and

$$
\begin{aligned}
& \frac{\mathrm{\ell}(\varepsilon)}{\mathrm{E}(\varepsilon)-\Xi}\left[{ }_{s}^{\psi} \mathcal{I}^{\theta, v}\left(\eta(\omega)-\Xi_{\zeta}(\omega)\right)^{z}\right]^{\frac{1}{z}} \\
& \quad \leq\left({ }_{s}^{\psi} \mathcal{I}^{\theta, v} \eta^{z}(\omega)\right)^{\frac{1}{z}} \leq \frac{\varphi(\varepsilon)}{\varphi(\varepsilon)-\Xi}\left[{ }_{s}^{\psi} \mathcal{I}^{\theta, v}\left(\eta(\omega)-\Xi_{\zeta}(\omega)\right)^{z}\right]^{\frac{1}{z}} .
\end{aligned}
$$


Multiplying both sides of (4.18) and (4.19) by $\frac{1}{v^{\theta} \Gamma(\theta)} \times \frac{\exp \left[\frac{v-1}{v}(\psi(\omega)-\psi(\varepsilon))\right]}{(\psi(\omega)-\psi(\varepsilon))^{1-\theta}} \psi^{\prime}(\varepsilon), \varepsilon<\omega$, then integrating the results with respect to $\varepsilon$ over $(s, \omega)$, we respectively obtain

$$
\begin{aligned}
& { }_{s}^{\psi} \mathcal{I}^{\theta, v}\left(\frac{1}{(\varphi(\omega)-\Xi)}\right)\left[{ }_{s}^{\psi} \mathcal{I}^{\theta, v}\left(\eta(\omega)-\Xi_{\zeta}(\omega)\right)^{z}\right]^{\frac{1}{z}} \\
& \leq \frac{(\psi(\omega)-\psi(s))^{\theta}}{v^{\theta} \Gamma(\theta+1)}\left({ }_{s}^{\psi} \mathcal{I}^{\theta, v} \varsigma^{z}(\omega)\right)^{\frac{1}{z}} \\
& \quad \leq{ }_{s}^{\psi} \mathcal{I}^{\theta, v}\left(\frac{1}{(\mathrm{E}(\omega)-\Xi)}\right)\left[{ }_{s}^{\psi} \mathcal{I}^{\theta, v}\left(\eta(\omega)-\Xi_{\zeta}(\omega)\right)^{z}\right]^{\frac{1}{z}}
\end{aligned}
$$

and

$$
\begin{aligned}
{ }_{s}^{\psi} \mathcal{I}^{\theta, v}\left(\frac{\mathrm{\complement}(\omega)}{\mathrm{E}(\omega)-\Xi}\right)\left[{ }_{s}^{\psi} \mathcal{I}^{\theta, v}\left(\eta(\omega)-\Xi_{\zeta}(\omega)\right)^{z}\right]^{\frac{1}{z}} \\
\leq \frac{(\psi(\omega)-\psi(s))^{\theta}}{v^{\theta} \Gamma(\theta+1)}\left({ }_{s}^{\psi} \mathcal{I}^{\theta, v} \eta^{z}(\omega)\right)^{\frac{1}{z}} \\
\quad \leq{ }_{s}^{\psi} \mathcal{I}^{\theta, v}\left(\frac{\varphi(\omega)}{\varphi(\omega)-\Xi}\right)\left[{ }_{s}^{\psi} \mathcal{I}^{\theta, v}\left(\eta(\omega)-\Xi_{\zeta}(\omega)\right)^{z}\right]^{\frac{1}{z}} .
\end{aligned}
$$

The addition of inequalities (4.20) and (4.21) completes the proof.

Theorem 4.3 Consider positive functions $\eta$, ऽ on $[0, \infty)$ with ${ }_{s}^{\psi} \mathcal{I}^{\theta, v} \eta^{z}(\omega),{ }_{s}^{\psi} \mathcal{I}^{\theta, v} \varsigma^{z}(\omega)<\infty$, $\forall \omega \in[0, \infty)$ and for all $v \in(0,1], \theta \in \mathbb{C}, \operatorname{Re}(\theta) \geq 0, z \geq 1$. Let $\psi$ be a strictly increasing continuousfunction. Assume that $\mathrm{E}, \varphi$ are two positive functions such that $0<\mathrm{七}(\kappa) \leq \frac{\eta(\kappa)}{\zeta(\kappa)} \leq$ $\varphi(\kappa), \kappa \in[s, \omega]$. Then we have

$$
\left({ }_{s}^{\psi} \mathcal{I}^{\theta, v} \eta^{z}(\omega)\right)^{\frac{1}{z}}+\left({ }_{s}^{\psi} \mathcal{I}^{\theta, v} \varsigma^{z}(\omega)\right)^{\frac{1}{z}} \leq 2\left({ }_{s}^{\psi} \mathcal{I}^{\theta, v} \xi^{z}(\eta(\omega), \varsigma(\omega))\right)^{\frac{1}{z}},
$$

where $\xi$ is an integrable function defined on $[0, \infty)$ by

$$
\xi(\eta(\omega), \varsigma(\omega))=\max \left\{\left[\left(1+\frac{\varphi(\omega)}{\mathrm{\complement}(\omega)}\right) \eta(\omega)-\varphi(\omega) \varsigma(\omega)\right], \frac{(\varphi(\omega)+\succeq(\omega)) \varsigma(\omega)-\eta(\omega)}{£(\omega)}\right\} .
$$

Proof Due to the assumption $0<\mathrm{七}(\kappa) \leq \frac{\eta(\kappa)}{\zeta(\kappa)} \leq \varphi(\kappa), \kappa \in[s, \omega]$, we get

$$
€(\kappa) \leq \varphi(\kappa)+\mathrm{Ł}(\kappa)-\frac{\eta(\kappa)}{\varsigma(\kappa)}
$$

and

$$
\varphi(\kappa)+\mathrm{七}(\kappa)-\frac{\eta(\kappa)}{\varsigma(\kappa)} \leq \varphi(\kappa) .
$$

By using the inequalities (4.23) and (4.24), we can write

$$
\varsigma(\kappa) \leq \frac{(\varphi(\kappa)+\succeq(\kappa)) \varsigma(\kappa)-\eta(\kappa)}{Ł(\kappa)} \leq \xi(\eta(\kappa), \varsigma(\kappa)),
$$

where $\xi$ is an integrable function defined on $[0, \infty)$ by

$$
\xi(\eta(\kappa), \varsigma(\kappa))=\max \left\{\left[\left(1+\frac{\varphi(\kappa)}{\mathrm{Ł}(\kappa)}\right) \eta(\kappa)-\varphi(\kappa) \varsigma(\kappa)\right], \frac{(\varphi(\kappa)+\succeq(\kappa)) \varsigma(\kappa)-\eta(\kappa)}{\mathrm{L}(\kappa)}\right\},
$$


which leads to

$$
\varsigma^{z}(\kappa) \leq \xi^{z}(\eta(\kappa), \varsigma(\kappa))
$$

On the other hand, from the assumption $\frac{1}{\varphi(\kappa)} \leq \frac{\zeta(\kappa)}{\eta(\kappa)} \leq \frac{1}{\epsilon(\kappa)}$, we have

$$
\frac{1}{\varphi(\kappa)} \leq \frac{1}{\varphi(\kappa)}+\frac{1}{\mathrm{Ł}(\kappa)}-\frac{\varsigma(\kappa)}{\eta(\kappa)}
$$

and

$$
\frac{1}{\varphi(\kappa)}+\frac{1}{€(\kappa)}-\frac{\varsigma(\kappa)}{\eta(\kappa)} \leq \frac{1}{€(\kappa)} .
$$

Using (4.27) and (4.28), we obtain

$$
\frac{1}{\varphi(\kappa)} \leq \frac{\left(\frac{1}{\varphi(\kappa)}+\frac{1}{\mathrm{E}(\kappa)}\right) \eta(\kappa)-\varsigma(\kappa)}{\eta(\kappa)} \leq \frac{1}{\mathrm{E}(\kappa)} .
$$

So

$$
\begin{aligned}
\eta(\kappa) & \leq \varphi(\kappa)\left(\frac{1}{v(\tau)}+\frac{1}{\mathrm{\complement}(\kappa)}\right) \eta(\kappa)-\varphi(\kappa) \varsigma(\kappa) \\
& =\left(1+\frac{\varphi(\kappa)}{\mathrm{七}(\kappa)}\right) \eta(\kappa)-\varphi(\kappa) \varsigma(\kappa) \\
& \leq \max \left\{\left[\left(1+\frac{\varphi(\kappa)}{\mathrm{七}(\kappa)}\right) \eta(\kappa)-\varphi(\kappa) \varsigma(\kappa)\right], \frac{(\varphi(\kappa)+\mathrm{\complement}(\kappa)) \zeta(\kappa)-\eta(\kappa)}{\mathrm{Ł}(\kappa)}\right\} \\
& =\xi(\eta(\kappa), \varsigma(\kappa)),
\end{aligned}
$$

which yields

$$
\eta^{z}(\kappa) \leq \xi^{z}(\eta(\kappa), \varsigma(\kappa))
$$

Multiplying both sides of (4.26) and (4.29) by $\frac{1}{v^{\theta} \Gamma(\theta)} \times \frac{\exp \left[\frac{\nu-1}{v}(\psi(\omega)-\psi(\kappa))\right]}{(\psi(\omega)-\psi(\kappa))^{1-\theta}} \psi^{\prime}(\kappa), \kappa<\omega$, then integrating the results with respect to $\kappa$ over $(s, \omega)$, we respectively get

$$
\left({ }_{s}^{\psi} \mathcal{I}^{\theta, v} \varsigma^{z}(\omega)\right)^{\frac{1}{z}} \leq\left({ }^{\psi} \mathcal{I}_{s}^{\theta, v} \xi^{z}(\eta(\omega), \varsigma(\omega))\right)^{\frac{1}{z}}
$$

and

$$
\left({ }_{s}^{\psi} \mathcal{I}^{\theta, v} \eta^{z}(\omega)\right)^{\frac{1}{z}} \leq\left({ }_{s}^{\psi} \mathcal{I}^{\theta, v} \xi^{z}(\eta(\omega), \varsigma(\omega))\right)^{\frac{1}{z}} .
$$

Hence, the addition of (4.30) and (4.31) ends the proof.

\section{Concluding remarks}

We have applied a proportional fractional integral operator with respect to another strictly increasing continuous function $\psi$. We then improved and generalized several consequences in the framework of fractional integral inequalities of Minkowski's type. The functions used in this work are bounded by two positive functions to get reverse Minkowski 
inequalities in the new setting. Moreover, we have introduced new fractional integral inequalities which have a close relationship to the reverse Minkowski-type inequalities via $\psi$-proportional fractional integral. Also with the aid of the considered operator, some new special cases of reverse Minkowski-type inequality have been discussed. The acquired results can be applied to emphasize the existence of nontrivial answers to various problems having fractional order. The results derived in this work are general in character and give some contributions to inequality theory, e.g., when $v$ and $\psi$ take different values, our results reduce to many results that include classical fractional operators such as RiemannLiouville, Hadamard, Katugampola, etc. As a future work, we are considering studying such results with respect to the Mittag-Leffler power law [41].

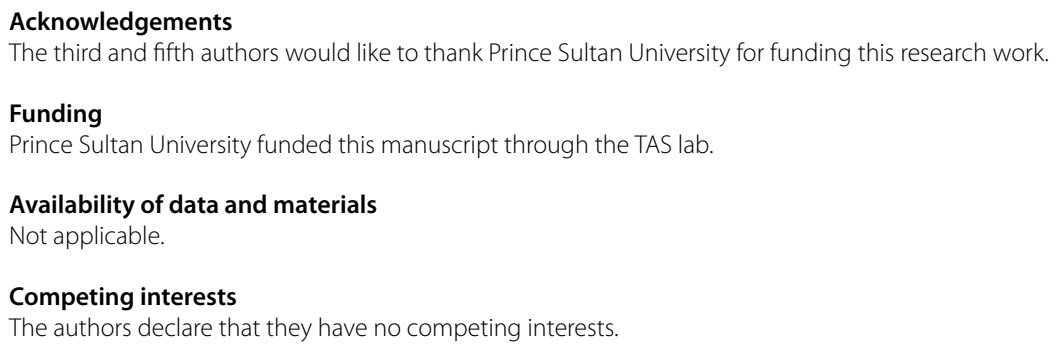

\section{Publisher's Note}

Springer Nature remains neutral with regard to jurisdictional claims in published maps and institutional affiliations.

Received: 29 May 2021 Accepted: 16 August 2021 Published online: 17 September 2021

\section{References}

1. Kilbas, A.A., Srivastava, H.M., Trujillo, J.J.: Theory and Applications of Fractional Differential Equations. North-Holland Mathematics Studies Elsevier, Amsterdam (2006)

2. Jarad, F., Abdeljawad, T.: Generalized fractional derivatives and Laplace transform. Discrete Contin. Dyn. Syst., Ser. S 13(3), 709-722 (2020)

3. Abdo, M.S., Shah, K., Panchal, S.K., Wahash, H.A.: Existence and Ulam stability results of a coupled system for terminal value problems involving $\psi$-Hilfer fractional operator. Adv. Differ. Equ. 2020, 316 (2020)

4. Abdo, M.S., Abdeljawad, T., Ali, S.M., Shah, K., Jarad, F.: Existence of positive solutions for weighted fractional order differential equations. Chaos Solitons Fractals 141, 110341 (2020). https://doi.org/10.1016/j.chaos.2020.110341

5. Wahash, H.A., Abdo, M.S., Saeed, A.M., Panchal, S.K.: Singular fractional differential equations with $\psi$-Caputo operator and modified Picard's iterative method. Appl. Math. E-Notes 20, 215-229 (2020)

6. Abdulwasaa, M.A., Abdo, M.S., Shah, K., Nofal, T.A., Panchal, S.K., Kawale, S.V., Abdel-Aty, A.H.: Fractal-fractional mathematical modeling and forecasting of new cases and deaths of COVID-19 epidemic outbreaks in India. Results Phys. 20, 103702 (2021)

7. Redhwan, S.S., Abdo, M.S., Shah, K., Abdeljawad, T., Dawood, S., Abdo, H.A., Shaikh, S.L.: Mathematical modeling for the outbreak of the coronavirus (COVID-19) under fractional nonlocal operator. Results Phys. 19, 103610 (2020)

8. Abdeljawad, T., Hajjib, M.A., Al-Mdallal, Q.M., Jarad, F.: Analysis of some generalized ABC-fractional logistic models. Alex. Eng. J. 59(4), 2141-2148 (2020)

9. Atangana, A.: Modelling the spread of COVID-19 with new fractal-fractional operators: can the lockdown save mankind before vaccination? Chaos Solitons Fractals 136, 109860 (2020)

10. Kreyszig, E.: Introductory Functional Analysis with Applications, vol. 1. Wiley, New York (1989)

11. Atangana, A., Araz, S.i.: Analysis of a new partial integro-differential equation with mixed fractional operators. Chaos Solitons Fractals 127, 257-271 (2019)

12. Araz, S.I.: Numerical analysis of a new Volterra integro-differential equation involving fractal-fractional operators. Chaos Solitons Fractals 130, Article ID 109396 (2020)

13. Atangana, A., Araz, S.I.: New concept in calculus: piecewise differential and integral operators. Chaos Solitons Fractals 145(1), Article ID 110638 (2021) 
14. Rahman, G., Abdeljawad, T., Jarad, F., Khan, A., Nisar, K.S.: Certain inequalities via generalized proportional Hadamard fractional integral operators. Adv. Differ. Equ. 2019, 454 (2019)

15. Rashid, S., Jarad, F., Noor, M.A., Kalsoom, H., Chu, Y.M.: Inequalities by means of generalized proportional fractional integral operators with respect another function. Mathematics 7(12), Article ID 1225 (2019)

16. Adjabi, Y., Jarad, F., Abdeljawad, T.: On generalized fractional operators and a Gronwall type inequality with applications. Filomat 31(17), 5457-5473 (2017)

17. Alzabut, J., Abdeljawad, T., Jarad, F., et al.: A Gronwall inequality via the generalized proportional fractional derivative with applications. J. Inequal. Appl. 2019, 101 (2019)

18. Bougoffa, L.: On Minkowski and Hardy integral inequalities. J. Inequal. Pure Appl. Math. 7(2), 60 (2006)

19. Dahmani, Z:: On Minkowski and Hermite-Hadamard integral inequalities via fractional integration. Ann. Funct. Anal. 1(1), 51-58 (2010)

20. Set, E., Ozdemir, M., Dragomir, S.: On the Hermite-Hadamard inequality and other integral inequalities involving two functions. J. Inequal. Appl. (2010). https://doi.org/10.1155/2010/148102

21. Yang, W: Some new fractional quantum integral inequalities. Appl. Math. Lett. 6(25), 963-969 (2012)

22. Taf, S., Brahim, K.: Some new results using Hadamard fractional integral. Int. J. Nonlinear Anal. Appl. 7(1), 103-109 (2015)

23. Chinchane, V.L., Pachpatte, D.B.: New fractional inequalities via Hadamard fractional integral. Int. J. Funct. Anal. Appl. 5(3), 165-176 (2013). https://doi.org/10.12785/msl/030301

24. da Vanterler, J., Sousa, C., Capelas de Oliveira, E.: The Minkowski's inequality by means of a generalized fractional integral. AIMS Ser. Appl. Math. 3(1), 131-147 (2018)

25. Rahman, G., Khan, A., Abdeljawad, T., Nisar, K.S.: The Minkowski inequalities via generalized proportional fractional integral operators. Adv. Differ. Equ. 2019, 287 (2019). https://doi.org/10.1186/s13662-019-2229-7

26. Aljaaidi, T.A., Pachpatte, D.B.: The Minkowski's inequalities via $\psi$-Riemann-Liouville fractional integral operators Rend. Circ. Mat. Palermo, II. Ser. (2020). https://doi.org/10.1007/s12215-020-00539-w

27. Rashid, S., Jarad, F., Chu, Y.: A note on reverse Minkowski inequality via generalized proportional fractional integral operator with respect to another function. Math. Probl. Eng. 2020, 7630260 (2020). https://doi.org/10.1155/2020/7630260

28. Maja, A., Farid, G., Pećarić, J., Siddique, U.: Generalized Minkowski type fractional inequalities involving extended Mittag-Leffler function. J. Indian Math. Soc. 87(3-4), 137-147 (2020)

29. Chinchane, V.L., Pachpatte, D.B.: New fractional inequalities involving Saigo fractional integral operator. Math. Sci. Lett. 3(3), 133-139 (2014)

30. Herrmann, R.: Fractional Calculus. An Introduction for Physicists. World Scientific, Singapore (2011)

31. Khan, H., Abdeljawad, T., Tunç, C., Alkhazzan, A., Khan, A.: Minkowski's inequality for the AB-fractional integral operator. J. Inequal. Appl. 2019, 96 (2019). https://doi.org/10.1186/s13660-019-2045-3

32. Mubeen, S., Habib, S., Naeem, M.N.: The Minkowski inequality involving generalized $k$-fractional conformable integral. J. Inequal. Appl. 2019, 81 (2019). https://doi.org/10.1186/s13660-019-2040-8

33. Sulaiman, W.T.: Reverses of Minkowski's, Hölder's, and Hardy's integral inequalities. Int. J. Mod. Math. Sci. 1(1), 14-24 (2012)

34. Usta, F., Budak, H., Ertugral, F., Sarıkaya, M.Z.: The Minkowski's inequalities utilizing newly defined generalized fractional integral operators. Commun. Fac. Sci. Univ. Ank. Sér. A1 Math. Stat. 68(1), 686-701 (2019)

35. Aljaaidi, T.A., Pachpatte, D.B.: Some Gruss-type inequalities using generalized Katugampola fractional integral. AIMS Math. 5(2), 1011-1024 (2020). https://doi.org/10.3934/math.2020070

36. Sousa, J., Oliveira, D.S., Capelas de Oliveira, E.: Gruss-type inequalities by means of generalized fractional integrals. Bull. Braz. Math. Soc. 50(4), 1029-1047 (2019). https://doi.org/10.1007/s00574-019-00138-z

37. Aljaaidi, T.A., Pachpatte, D.B.: New generalization of reverse Minkowski's inequality for fractional integral. Adv. Theory Nonlinear Anal. Appl. 5(1), 72-81 (2021). https://doi.org/10.31197/atnaa.756605

38. Samko, S.G., Kilbas, A.A., Marichev, O.l., et al.: Fractional Integrals and Derivatives. Theory and Applications. Gordon \& Breach, Yverdon (1993)

39. Jarad, F., Abdeljawad, T., Alzabut, J.: Generalized fractional derivatives generated by a class of local proportional derivatives. Eur. Phys. J. Spec. Top. 226, 3457-3471 (2017)

40. Jarad, F., Alqudah, M.A., Abdeljawad, T.: On more generalized form of proportional fractional operators. Open Math 18, 167-176 (2020)

41. Atangana, A., Baleanu, D.: New fractional derivatives with non-local and non-singular kernel: theory and application to heat transfer. Model. Therm. Sci. 20(2), 763-769 (2016)

\section{Submit your manuscript to a SpringerOpen ${ }^{\circ}$ journal and benefit from:}

- Convenient online submission

- Rigorous peer review

- Open access: articles freely available online

- High visibility within the field

- Retaining the copyright to your article

Submit your next manuscript at $>$ springeropen.com 